\title{
INFLUÊNCIA DOS ÓLEOS BÁSICOS NO DESEMPENHO DE LUBRIFICANTES DE MOTOR
}

\author{
Maristela de F. V. Melachus ${ }^{1}$, Roberta M. Teixeira ${ }^{1}$, Sergio L. C. Viscardi ${ }^{1}$ \\ ${ }^{1}$ Ipiranga Produtos de Petróleo S.A.
}

E-mails: maristelav@ipiranga.com.br, robertamt@ipiranga.com.br, viscardi@ipiranga.com.br

\section{RESUMO}

De acordo com a classificação API, os óleos básicos estão divididos em cinco grupos. Desses, três grupos são relacionados às bases minerais parafínicas (grupo I, II e III) tendo como parâmetro de diferenciação o teor de saturados, enxofre e índice de viscosidade. Além das diferenças de propriedades encontradas entre os três grupos API, é importante salientar que dentro de um mesmo grupo são ofertados básicos de qualidades distintas. Estas diferenças podem impactar diretamente na formulação do lubrificante no que se refere às propriedades físico-químicas e também em sua sinergia com os aditivos levando a diferentes níveis de desempenho na aplicação. O estudo realizado tem como objetivo avaliar o desempenho de produtos formulados com blend oils de diferentes origens pertencentes ao mesmo grupo API mantendo-se constante o pacote de aditivos e taxas de tratamento. Foram determinadas as propriedades de fluxo a frio, viscosimétricas, volatilidade, estabilidade térmica e oxidativa. Com base nos resultados obtidos, pode-se observar a complexidade da formulação de produtos de alto desempenho. A escolha do blend oil é essencial e deve sempre estar relacionada à sinergia com o pacote de aditivos para que possamos obter produtos de alto desempenho.

Palavras-chave: óleos básicos; classificação API; desempenho; sinergia;

\section{INTRODUÇÃO}

Os lubrificantes, em sua maioria, têm origem mineral, vegetal, animal e sintética. Os mais utilizados são os óleos minerais que são obtidos a partir do petróleo e dependendo de sua estrutura molecular são classificados em óleos básicos parafínicos, naftênicos e aromáticos. A estrutura está relacionada com a origem do petróleo e processo de obtenção. Cada tipo de óleo básico corresponde a uma série de propriedades desejáveis, conforme a aplicação do lubrificante [1].

De acordo com a classificação API, os óleos básicos estão divididos em cinco grupos que levam em conta a sua especificação em relação ao teor de saturados, teor de enxofre e índice de viscosidade (IV). Sendo que, três grupos são relacionados às bases minerais parafínicas (Grupo I, II e III) tendo como parâmetros de diferenciação o teor de saturados, teor de enxofre e o índice de viscosidade conforme Tabela 1 [1]. 
Tabela 1 - Classificação API para óleos básicos [1]

\begin{tabular}{|c|c|c|c|}
\hline Grupo & Saturados & Enxofre & IV \\
\hline I & $<90 \%$ & $>0,03 \%$ & $80<$ IV $<120$ \\
\hline II & $>90 \%$ & $<0,03 \%$ & $80<$ IV $<120$ \\
\hline III & $>90 \%$ & $<0,03 \%$ & IV $>120$ \\
\hline
\end{tabular}

Existem ainda outros grupos classificados pela API (IV e V), porém não serão abordados neste trabalho.

Além das diferenças de propriedades encontradas entre os três grupos API, é importante salientar que dentro de um mesmo grupo são ofertados óleos básicos de qualidades distintas. Estas diferenças podem impactar diretamente na formulação do lubrificante no que se refere às propriedades físico-químicas e também em sua sinergia com os aditivos levando a diferentes níveis de desempenho na aplicação. Por este motivo, torna-se essencial o entendimento relacionado aos diferentes conceitos de especificação e desempenho.

Especificação é a faixa de variação de cada uma das características do óleo básico que permitem identificá-lo. Esta identificação é realizada através de análises típicas que visam à caracterização do produto. Entretanto, convém ressaltar que dois produtos que possuem a mesma análise típica podem não apresentar o mesmo desempenho no lubrificante formulado.

Desempenho é o comportamento do lubrificante numa dada aplicação o qual é verificado por meio de testes específicos. Nestas aplicações, os aditivos são os principais responsáveis pelo desempenho do lubrificante, porém torna-se essencial a definição adequada do blend oil, o qual em sinergia com os pacotes de aditivos proporcionam o atendimento aos padrões de desempenho exigidos pelos principais institutos, como API e ACEA, bem como OEMs.

Com base no exposto, o estudo a seguir tem como principal objetivo avaliar o desempenho de produtos formulados com blend oils de diferentes origens pertencentes ao mesmo grupo API mantendo-se constante o pacote de aditivos e taxas de tratamento.

\section{METODOLOGIA}

Foi conduzido um estudo comparativo entre diferentes formulações de três óleos lubrificantes, onde a variável estudada foi o blend oil, desta forma os pacotes de aditivos e taxas de tratamento foram mantidos para cada grau SAE e nível de desempenho API, totalizando 11 amostras avaliadas. O detalhamento das formulações preparadas pode ser visualizado na Tabela 2. 
Tabela 2 - Óleos Lubrificantes preparados com óleos básicos de diferentes origens

\begin{tabular}{|c|c|c|}
\hline Grupo & Identificação & Blend Oil \\
\hline \multirow{4}{*}{ 15W40 SL } \\
\cline { 2 - 3 } & Grupo I (A) & $150+500$ \\
\cline { 2 - 3 } & Grupo I (B) & $150+500$ \\
\cline { 2 - 3 } & Grupo I (C) & $150+600$ \\
\hline \multirow{4}{*}{ 15W40 SL } & Grupo I (D) & $150+500$ \\
\cline { 2 - 3 } & Grupo II (A) & $220(\mathrm{~B})+220(\mathrm{C})$ \\
\cline { 2 - 3 } & Grupo II (B) & 220 \\
\cline { 2 - 3 } & Grupo II (C) & 170 \\
\hline \multirow{3}{*}{ 5W30 SN } & Grupo II (D) & $4+6$ \\
\cline { 2 - 3 } & Grupo III (A) & $4+6$ \\
\cline { 2 - 3 } & Grupo III (B) & $4+6$ \\
\hline
\end{tabular}

*Identificação das viscosidades dos básicos utilizados nas misturas, os quais são de origens diferentes: GI e GII (SSU), GIII (cSt)

Para avaliar o impacto da escolha do blend oil no desempenho dos lubrificantes formulados foram determinadas as propriedades de fluxo a frio, estabilidade térmica e oxidativa, volatilidade, bem como as viscosimétricas. A Tabela 3 apresenta os ensaios realizados e respectivas normas de referência.

Tabela 3 - Ensaios comparativos realizados nas amostras de lubrificantes

$\left.\begin{array}{|c|c|}\hline \text { Ensaio } & \text { Norma referência } \\ \hline \text { Termogravimetria (TGA) } & {[2],[3],[4]} \\ \hline \text { Estabilidade oxidativa - RPVOT } & \text { ASTM D5481, [5] } \\ \hline \text { Viscosidade a alta temperatura e alta pressão - HT/HS } & \text { ASTM D2272, [6] } \\ \hline \begin{array}{c}\text { Determinação da tensão de escoamento e viscosidade } \\ \text { aparente de óleos de motor a baixa temperatura - MRV }\end{array} & \text { ASTM D4684, [7] } \\ \hline \begin{array}{c}\text { Determinação da viscosidade aparente de óleos de motor } \\ \text { entre -5 e -35 }{ }^{\circ} \text { C usando o simulador a frio - CCS }\end{array} & \text { ASTM D5800 (método B), [9] } \\ \hline \begin{array}{c}\text { Determinação da perda por evaporação de óleos } \\ \text { lubrificantes - NOACK }\end{array} & \text { ASTM D6186, [10], [11], [12], } \\ \hline \text { Calorimetria Diferencial Exploratória - PDSC }\end{array}\right]$

\section{RESULTADOS}

A Estabilidade oxidativa indica a capacidade de resistência à oxidação do óleo quando submetido a longos períodos de estocagem ou sob condições dinâmicas de uso.

As figuras 1 e 2 apresentam o comparativo entre os resultados das amostras do lubrificante SAE 15W40 SL formulado com os grupos I e II nos ensaios de estabilidade oxidativa medidos por bomba rotatória (RPVOT) e por PDSC, respectivamente. É possível verificar que as amostras formuladas com blend oil grupo II apresentaram um desempenho superior se comparado às formuladas com o grupo I pelas duas técnicas. Analisando cada grupo 
individualmente, as amostras grupo I (D) e grupo II (C) se destacam no ensaio de RPVOT enquanto que na análise por PDSC o desempenho das amostras grupo I (C), grupo II (C) e (D) se destacam das demais. Porém a amostra de óleo básico 150 utilizada na formulação grupo I (D) apresentou presença de antioxidante, o que descaracteriza o resultado para efeitos de comparação. O resultado da amostra grupo I (B) não foi reportado, pois a mesma não apresentou evento para o equipamento PDSC. Acredita-se que a amostra sofreu oxidação em temperatura inferior ao estipulado no método na qual as outras amostras foram analisadas.
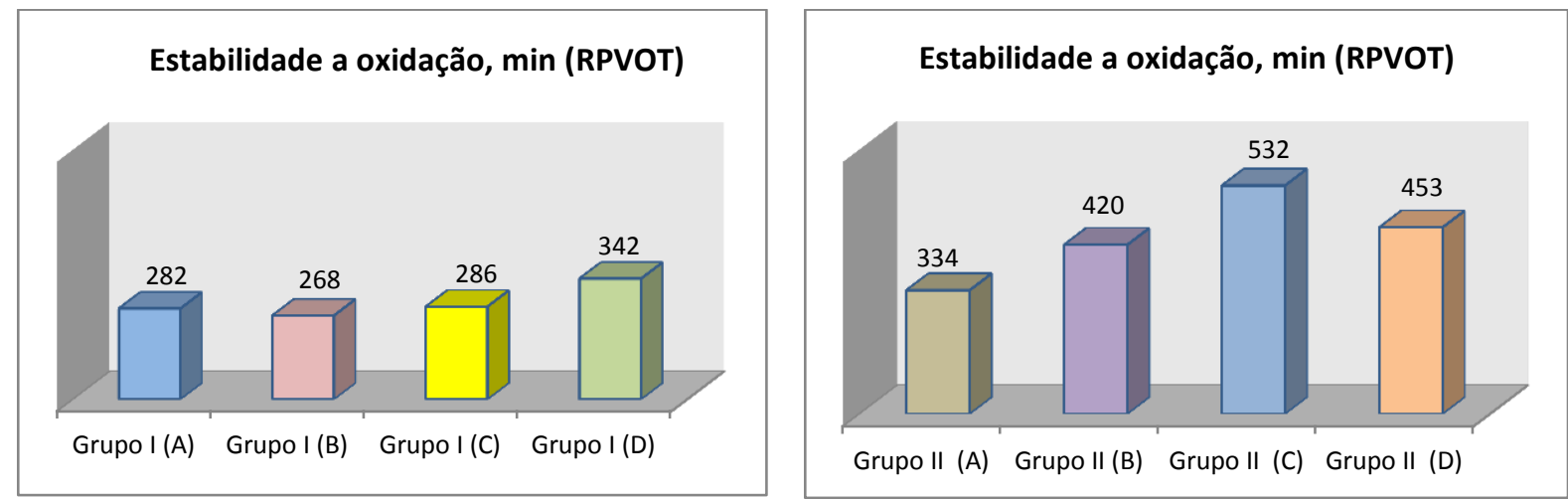

Figura 1 - Estabilidade a oxidação (RPVOT), tempo (min) - 15W40 SL
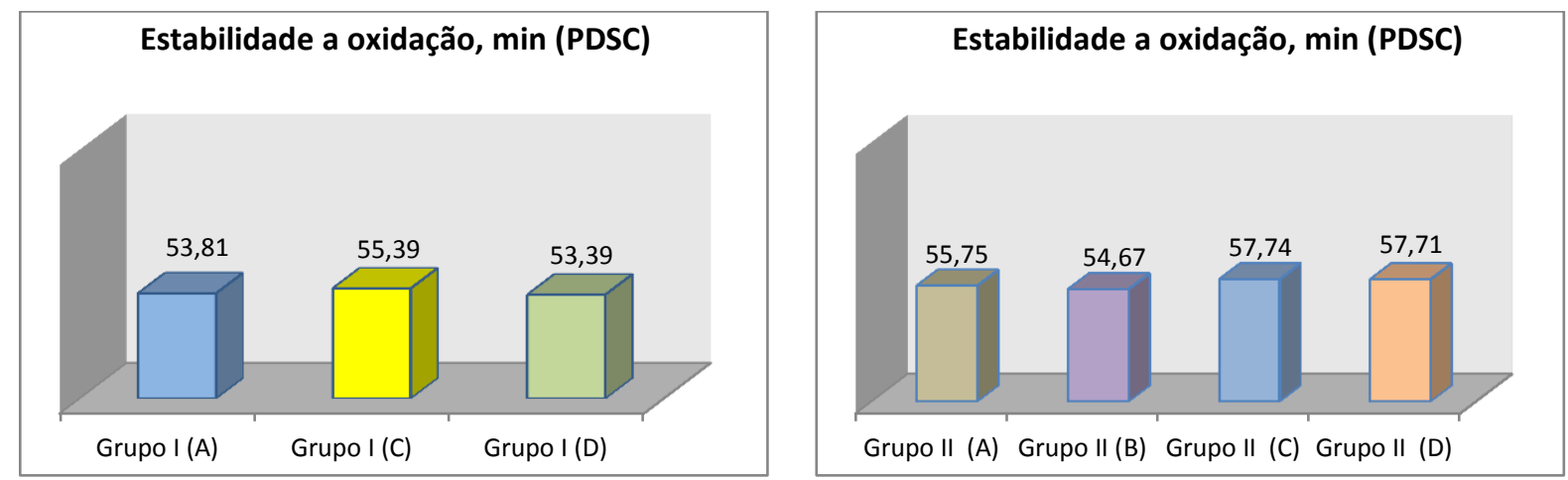

Figura 2 - Estabilidade a oxidação (PDSC), tempo (min) - 15W40 SL

A Figura 3 apresenta o comparativo entre as amostras do lubrificante SAE 5W30 SN formulado com básicos do grupo III nos ensaios de RPVOT e PDSC, respectivamente. Observa-se que a amostra grupo III (B) apresentou menor estabilidade oxidativa nas tuas técnicas avaliadas.
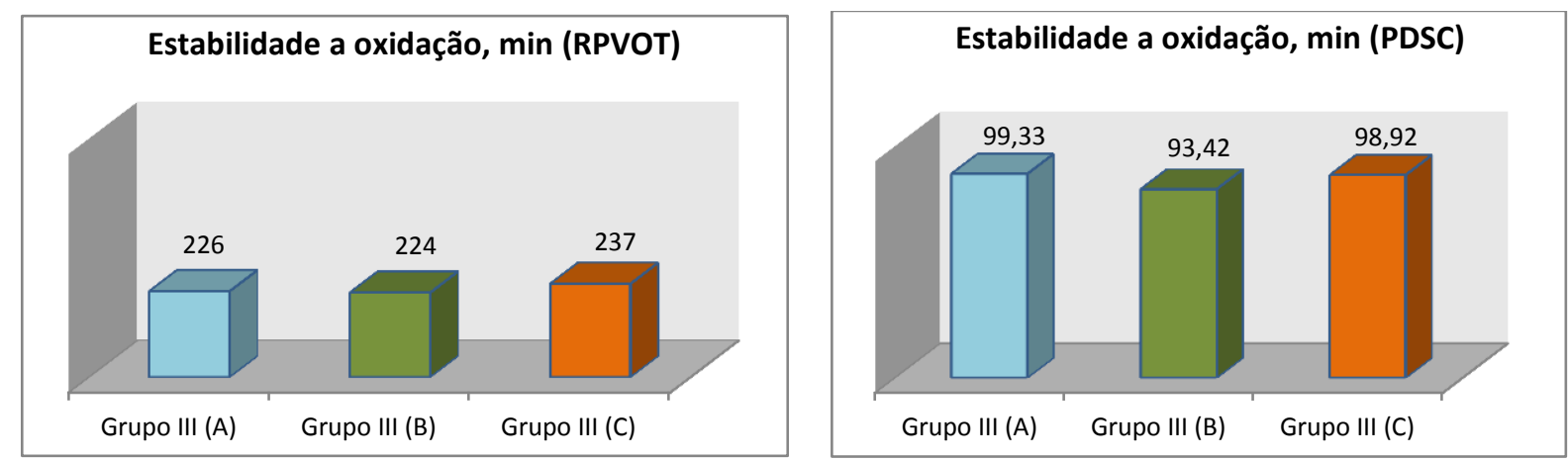

Figura 3 - Estabilidade a oxidação, min (RPVOT) e (PDSC) - 5W30 SN 
A seguir são apresentados os gráficos com os resultados obtidos na análise termogravimétrica (TGA) que avalia o processo de degradação térmica de lubrificantes. Essa técnica permite avaliar a estabilidade térmica de um material com base na variação de massa por evaporação ou decomposição, em função da temperatura em uma atmosfera controlada. Essa perda de massa pode dar-se devido à volatilização de componentes mais leves, bem como a oxidação da amostra quando em atmosfera de oxigênio. A medida é denominada onset de temperatura $\left({ }^{\circ} \mathrm{C}\right)$, a qual é definida como a temperatura inicial de perda de massa da amostra nas condições estudadas.

A Figura 4 apresenta os resultados para as formulações de lubrificante $15 \mathrm{~W} 40$ SL. Podemos verificar que as amostras do grupo II apresentam melhor desempenho se comparadas às amostras do grupo I. Quando avaliamos os grupos individualmente, observamos que a amostra grupo I (D) possui uma melhor estabilidade térmica enquanto as amostras de grupo II apresentaram resultados semelhantes. Esta propriedade sofre grande interferência, mas não somente, da volatilidade do óleo, ou seja, quanto mais volátil menor o onset de temperatura.
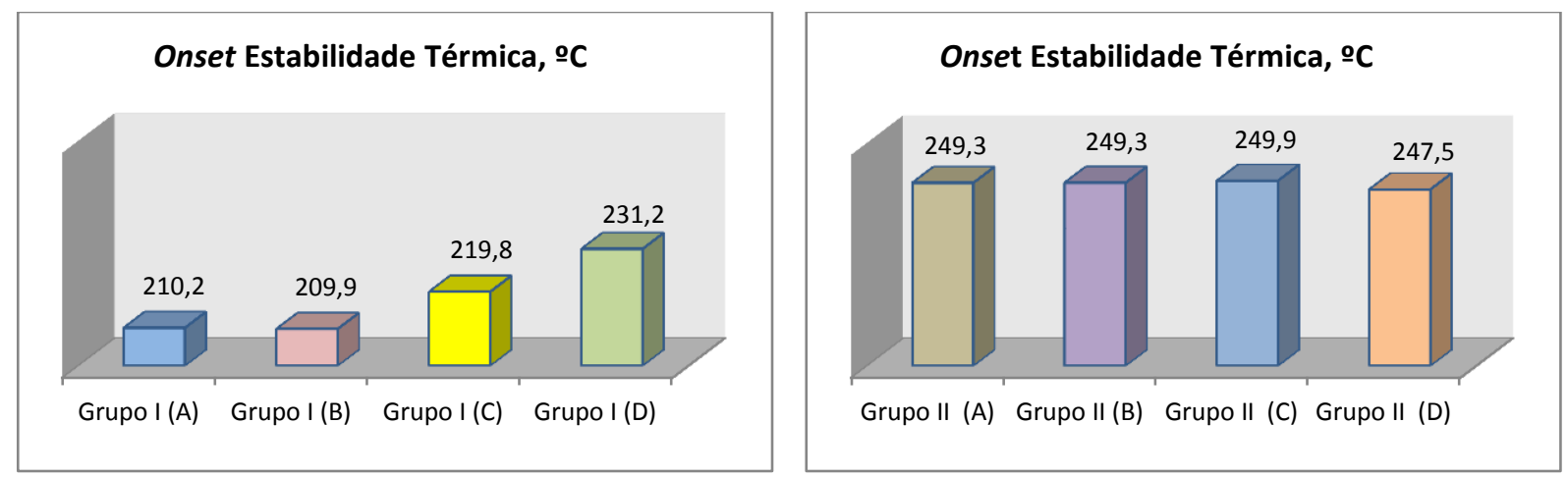

Figura 4 - Onset Estabilidade térmica $\left({ }^{\circ} \mathrm{C}\right)-15 \mathrm{~W} 40 \mathrm{SL}$

A figura 5 nos mostra o comparativo entre as amostras do lubrificante SAE 5W30 SN formulado com básicos do grupo III, podemos verificar diferenças consideráveis entre as três misturas, mesmo sendo formuladas com óleos básicos de viscosidade semelhante. A amostra grupo III (C) apresentou melhor desempenho nesta propriedade.

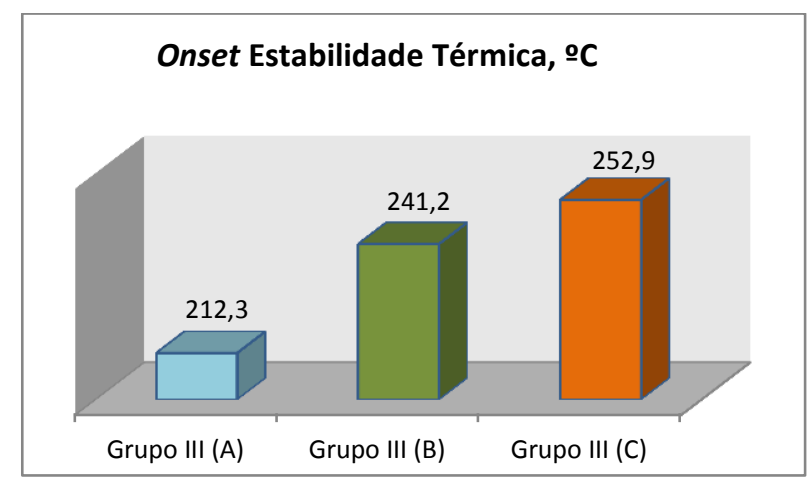

Figura 5 - Onset Estabilidade térmica $\left({ }^{\circ} \mathrm{C}\right)-5 \mathrm{~W} 30$ SN

As Figuras 6 e 7 apresentam são os resultados obtidos nas análises de perda por evaporação (Noack), para as formulações de 15W40 SL e 5W30 SN, respectivamente. O teste em questão avalia as perdas dos hidrocarbonetos mais leves do óleo quando submetido a temperaturas elevadas, o que levaria ao maior consumo do óleo e alteração de suas características. Essa propriedade está relacionada à perda de nível do lubrificante em serviço. 
Em concordância com a análise termogravimétrica, ao comparar as amostras do óleo lubrificante $15 \mathrm{~W} 40$ SL formulado com básico grupo II, é possível verificar que apresentam menor perda por evaporação, contribuindo para uma maior estabilidade térmica. $\mathrm{Na}$ comparação do óleo lubrificante $5 \mathrm{~W} 30 \mathrm{SN}$, verificamos que a amostra grupo III (A) apresenta perda por evaporação superior. Podemos correlacionar este resultado com a análise termogravimétrica, onde a mesma amostra apresentou estabilidade térmica inferior a das demais amostras do mesmo grupo.
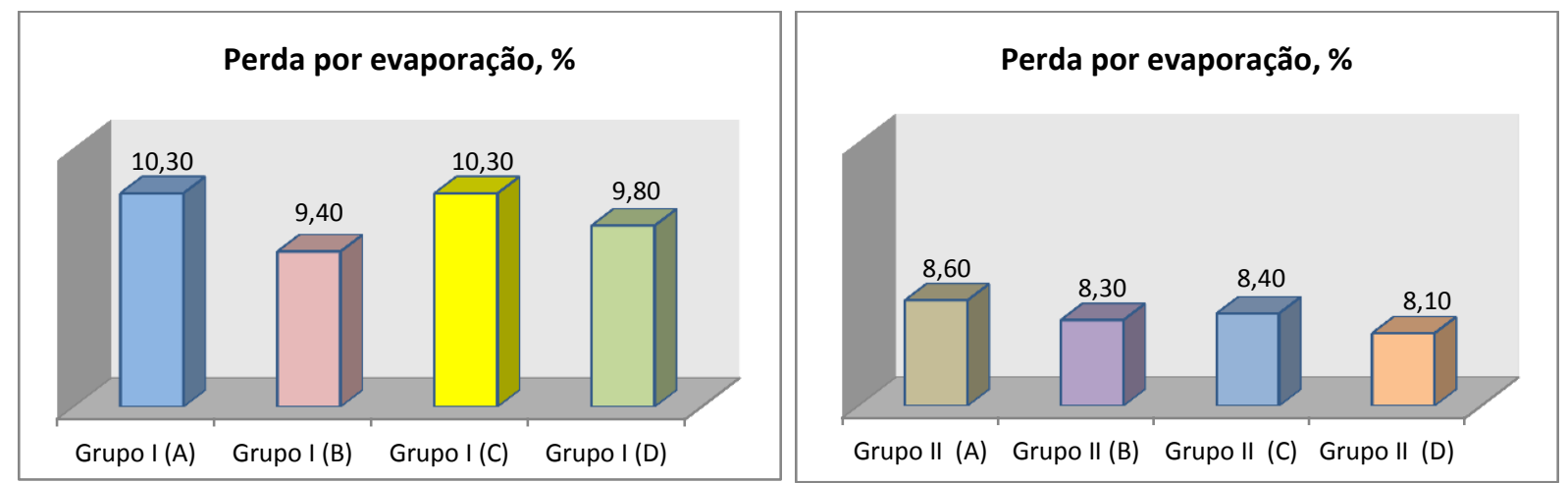

Figura 6 - Perda por evaporação, \% - 15W40 SL

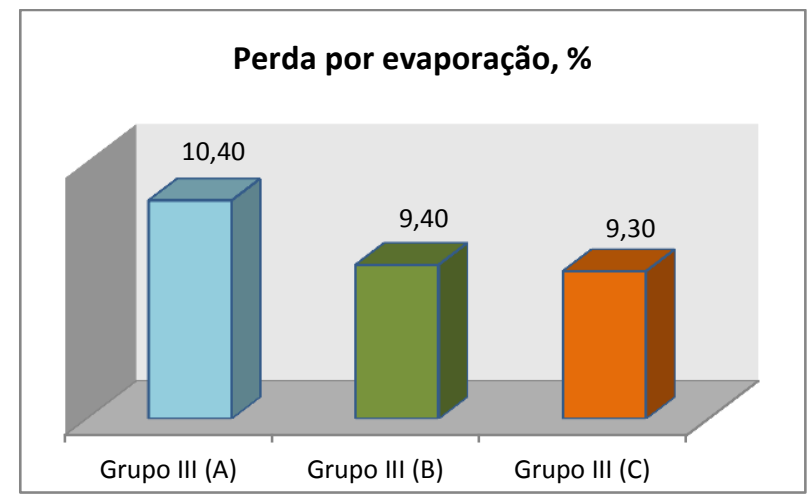

Figura 7 - Perda por evaporação, \% - 5W30 SN

As Figuras 8 e 9 apresentam os resultados de MRV para as formulações de 15W40 SL e 5W30 SN, respectivamente. Este ensaio determina a tensão de escoamento e viscosidade aparente de óleos de motor a baixa temperatura. Nesse ensaio, avaliamos a bombeabilidade do óleo a baixas temperaturas. Ou seja, quanto menor a viscosidade aparente, melhor desempenho terá o lubrificante.

Ao comparar os resultados da Figura 8, verificamos que as amostras do grupo II apresentaram maior desempenho se comparadas às amostras do grupo I para as formulações 15W40 SL. Analisando cada grupo individualmente, observamos que as amostras grupo I (B) e grupo II (B) têm desempenho inferior. 

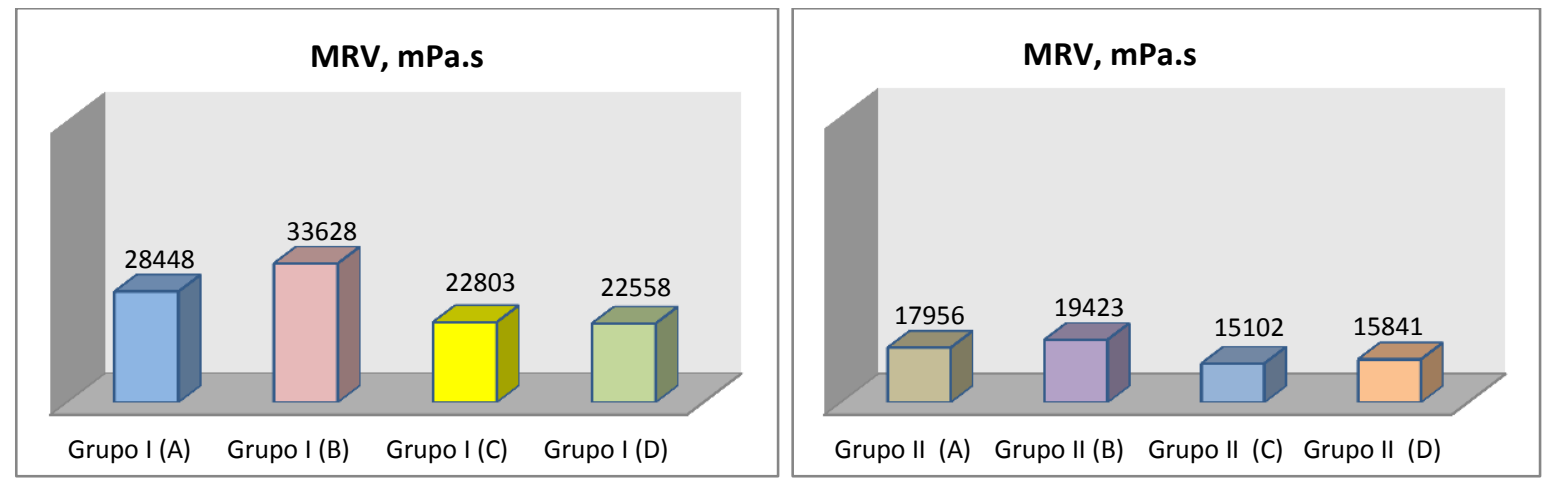

Figura 8 - MRV (Mini Rotary Viscometer), mPa.s - 15W40 SL

Na Figura 9 observamos que a amostra grupo III (B) possui um desempenho inferior em relação às amostras do grupo 5W30 SN.

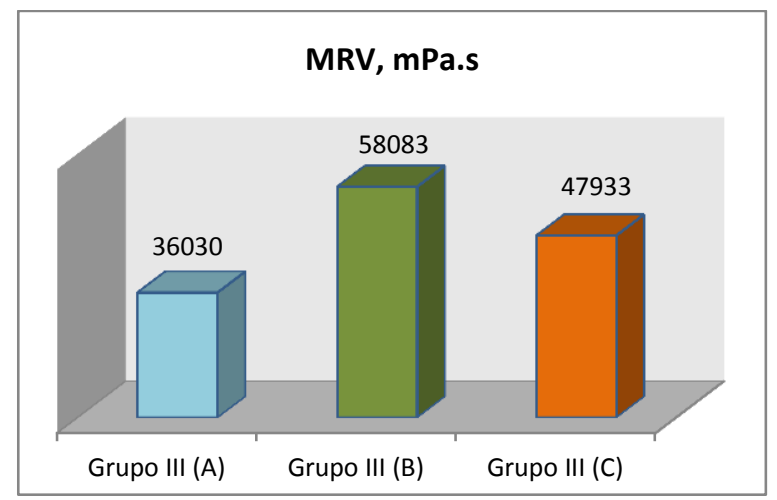

Figura 9-MRV (Mini Rotary Viscometer), mPa.s - 5W30 SN

As Figuras 10 e 11 apresentam os resultados obtidos no simulador de partida a frio (CCS Cold Cranking Simulator) para as amostras de 15W40 SL e 5W30 SN, respectivamente. O ensaio determina a viscosidade aparente, operando em uma faixa de temperatura de $0^{\circ} \mathrm{C} \mathrm{a}$ $40^{\circ} \mathrm{C}$. Tal método tem demonstrado uma excelente correlação com os dados práticos em motores e é utilizado pela classificação SAE J300 para determinar os limites de viscosidade dos óleos de motor multiviscosos e também dos lubrificantes que atendem às especificações de baixas temperaturas.

Com base na Figura 10, observamos que a amostra que utilizou o grupo II (D) apresentou desempenho superior, enquanto que a amostra grupo I (B) apresentou desempenho inferior nos respectivos grupos. Em concordância com os resultados obtidos por MRV, se compararmos as amostras formuladas com blend oils dos grupos I e II, verificamos que o grupo II possui melhor desempenho na partida a frio. 

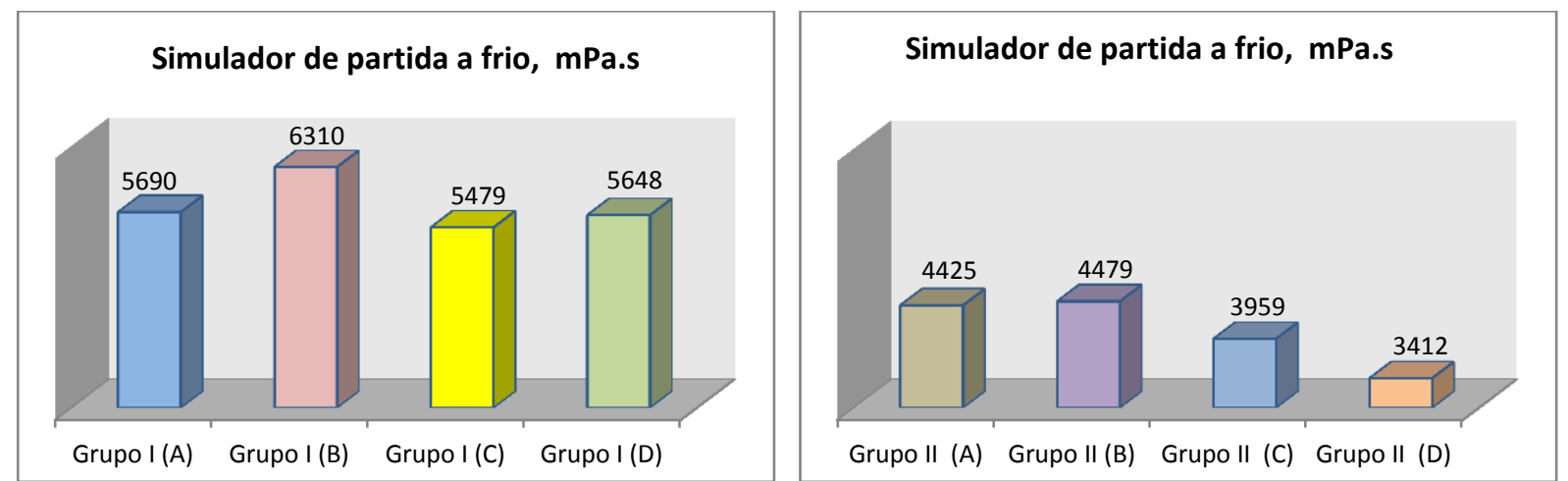

Figura 10 - Simulador de partida a frio, mPa.s - 15W40 SL

Observando-se os resultados expressos na Figura 11, verificamos que a amostra que utilizou o grupo III (C) apresentou desempenho superior.

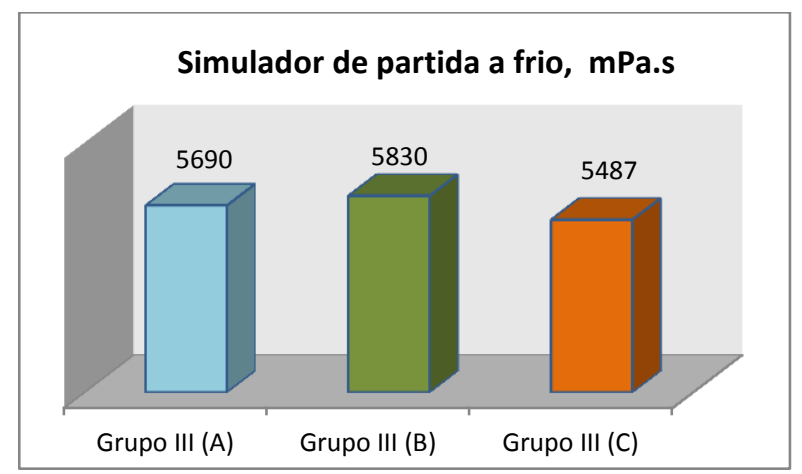

Figura 11 - Simulador de partida a frio, mPa.s - 5W30 SN

As Figuras 12 e 13 reportam os resultados encontrados na determinação da viscosidade à alta temperatura e alta pressão (HTHS - High Temperature High Shear) para as amostras de 15W40 SL e 5W30 SN, respectivamente. Embora os resultados não tenham apresentado diferença significativa, há uma tendência para as amostras que apresentaram viscosidades maiores, demonstrarem resultados de HT/HS superiores.
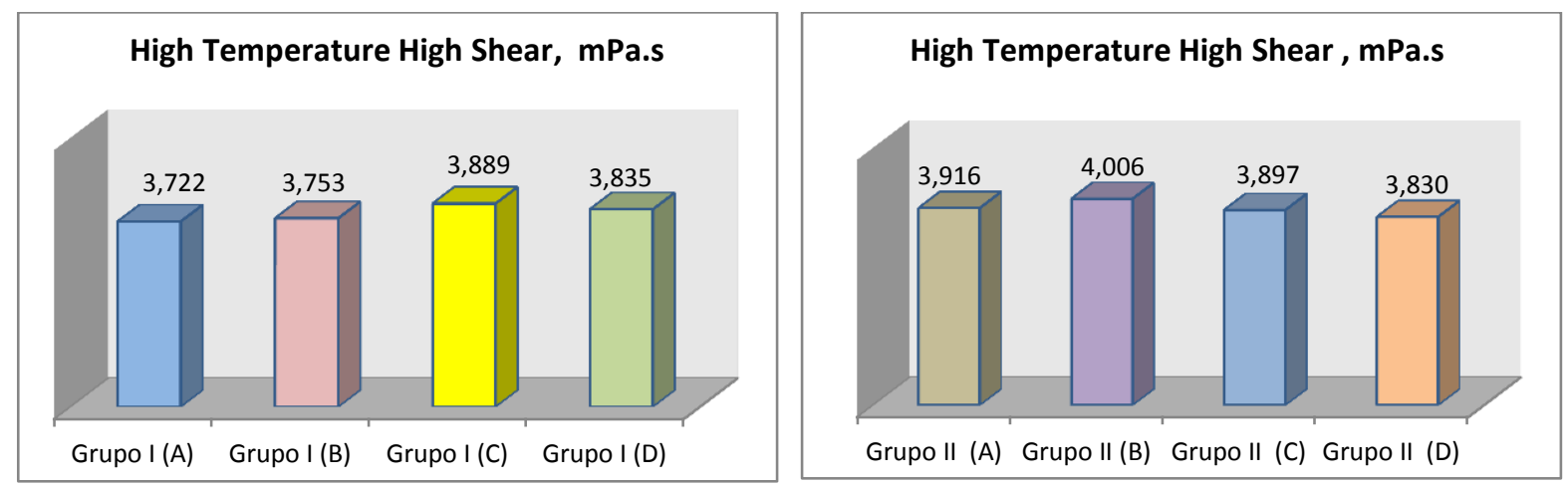

Figura $12-\mathrm{HT} / \mathrm{HS}, \mathrm{mPa} . \mathrm{s}-15 \mathrm{~W} 40 \mathrm{SL}$ 


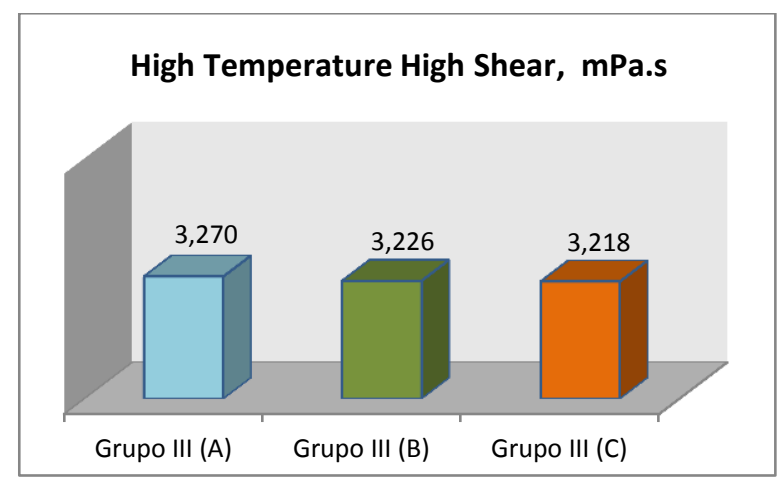

Figura $13-\mathrm{HT} / \mathrm{HS}, \mathrm{mPa} . \mathrm{s}-5 \mathrm{~W} 30 \mathrm{SN}$

\section{CONCLUSÃO}

Para a formulação do lubrificante 15W40 SL, as amostras que utilizaram óleos básicos do grupo II apresentaram melhor desempenho quando comparadas as formulações com grupo I. Porém comparando-se as amostras formuladas com básicos do mesmo grupo API foi possível verificar que as mesmas apresentaram diferente desempenho para um mesmo pacote de aditivos.

De uma forma geral as amostras C e D do grupo II e C do grupo I apresentaram melhores resultados.

Observação semelhante pode ser realizada nas amostras de lubrificante $5 \mathrm{~W} 30 \mathrm{SN}$, as quais embora tenham sido formuladas apenas com óleos básicos do mesmo grupo API (III), o blend oil (C) apresentou o melhor desempenho nas propriedades estudadas quando comparado aos demais.

Com base nos resultados obtidos podemos mostrar a complexidade de formulação de um produto de alto desempenho, em que as análises físico-químicas são somente o início de um processo. A escolha do blend oil é importantíssima e deve sempre estar relacionada à sinergia com o pacote de aditivos para que possamos atingir a produção de produtos de alto desempenho. Cabe salientar que o desempenho de um óleo lubrificante deve ser comprovado após sua aplicação e avaliação em testes de motor.

\section{REFERÊNCIAS}

[1] Carreteiro, R.P., Belmiro P.N.A., Lubrificantes \& Lubrificação Industrial. Editora Iterciência, Rio de Janeiro - 2006;

[2] GAMLIN C. D., DUTTA N.K., CHOUDHURY N. Roy, KEHOE D., MATISONS J. Evaluation of Kinetic parameters of thermal and oxidative decomposition of base oils by conventional, isothermal and modulated TGA, and pressure DSC. Thermochimica Acta 392393, 357-369, 2002;

[3] PEREZ Joseph. Oxidative properties of lubricants using thermal analysis. Thermochimica Acta 357-358, 47-56, 2000;

[4] BARMAN B.N. Behavioral differences between group I and group II base oils during thermo-oxidative degradation. Tribology International 35, 15-26, 2002; 
[5] ASTM D5481 - 13 - Standard Test Method for measuring Apparent Viscosity at HighTemperature and High-Shear Rate by Multicell Capillary Viscometer;

[6] ASTM D2272 - 14a - Standard Test Method for Oxidation Stability of Steam turbine Oils by Rotating Pressure Vessel;

[7] ASTM D4684 - 2014 - Standard Test Method for Determination of Yield Stress and Apparent Viscosity of Engine Oils at Low Temperature;

[8] ASTM D5293 - 2014 - Standard Test Method for Apparent Viscosity of Engine Oils and Base Stocks between $-5^{\circ} \mathrm{C}$ and $-35^{\circ} \mathrm{C}$ Using Cold-Craking Simulator;

[9] ASTM D5800 (método B) - 2015 - Standard Test Method for Evaporation Loss of Lubricating Oils by the Noack Method;

[10] ASTM D6186 - 2008 - Standard Test Method for Oxidation Induction Time of Lubricating Oils by Pressure Differential Scanning Calorimetry;

[11] ADHVARYU A., ERHAN S.Z., SAHOO S.K., SINGH I.D. Thermo-oxidative stability studies on some new generation API group II and III base oils. Fuel 81, 785-791, 2002;

[12] JAROSLAV Cerny, MIROSLAV Zelinka. Oxidation Stability of lubricants measured by a PDSC Technique. Petroleum \& Coal 46 (3), 56-62, 2004;

[13] ADAMCZEWSKA Jolanta. Oxidative Stability of lubricants by PDSC CEC L-85T-99

Test Procedure. Journal of Thermal Analysis and Calorimetry, Vol. 80, 753-759, 2005; 